\title{
AUTOMATIC SERVICE COMPOSITION VIA SIMULATION
}

\author{
DANIELA BERARDI ${ }^{1}$, FAHIMA CHEIKH ${ }^{2}$, GIUSEPPE DE GIACOMO ${ }^{1}$, FABIO PATRIZI ${ }^{1}$ \\ 1 Dipartimento di Informatica e Sistemistica, Università di Roma "La Sapienza" \\ Via Salaria 113, 00198 Roma, Italy \\ lastname@dis. uniroma1.it \\ 2 Université Paul Sabatier, Institut de Recherche en Informatique de Toulouse \\ 31062 Toulouse Cedex 9, France \\ cheikh@irit. fr \\ Received (received date) \\ Revised (revised date) \\ Communicated by Editor's name
}

\section{ABSTRACT}

In this paper we study the issue of service composition, for services that export a representation of their behavior in the form of a finite deterministic transition system. In particular, given a specification of the target service requested by the client as a finite deterministic transition system, the problem we face is how we can exploit the computations of the available services for realizing the computations of the target service. While ways to tackle such a problem are known, in this paper we present a new technique that is based on the notion of simulation, which is still optimal from the computational complexity point. Notably, such a technique, opens up the possibility of devising composition in a "just-in-time" fashion. Indeed, we show that, by exploiting simulation, it is actually possible to implicitly compute all possible compositions at once, and delay the choice of the actual composition to run-time.

\section{Introduction}

Service Oriented Computing (SOC) is the computing paradigm that utilizes services as fundamental elements for realizing distributed applications/solutions. Services are self-describing, platform-agnostic computational elements that are advocated to support rapid, low-cost and easy composition of loosely coupled distributed applications $[2,36,24]$. From a practical point of view, services are modular applications that can be described, published, located and invoked over a network: any piece of code and any application component deployed on a system can be wrapped and transformed into a network-available service. Interestingly, description of services are quite high level: typically, services -or, better said, the computations they provide- are described in terms of finite state transition systems [21].

The availability of high level descriptions of the computations provided by a service opens the possibility of composing services in an automatic way, with the aim of realizing target computations. Indeed, while formal analysis and synthesis 
of full fledged programs is still considered prohibitive, once we focus on high level descriptions of the programs several verification and synthesis techniques developed in various areas of Computer Science become exploitable for automatic composition of services. As a result automatic service composition has been investigated in several contexts: services seen as atomic actions, e.g., [1], by relying on AI Planning research [16]; services seen as information providers, e.g., [25], by relying on data integration work [34, 18, 22]; services seen as complex processes that can engage in a variety of conversations, e.g., $[28,23,9,7]$, by relying, at least implicitly, on the literature on process synthesis $[29,35,33]$.

In this paper, we look at the latter context. In particular, we look at one of the most intriguing service composition proposals, known as the Roman Model [5, 7]. In such a proposal, available services are characterized by their conversational behavior, compactly represented as finite transition systems. The goal of the composition task is to realize a new service specified by the client -again, as a finite transition system- by combining those computation fragments the available services provide.

In other words, the Roman Model envisions a kind of "service integration system" which makes available a pool of virtual building blocks to clients. By making use of such virtual blocks, a client can write its own service as a high-level program, abstractly represented by a finite transition system. Actually, virtual blocks are not implemented directly, but made available through the service composition. The actual services available to the system are themselves formally described as high level programs, built out of such virtual blocks. Such a description can be considered as a mapping from concrete service to virtual blocks of the integration system. The idea is to exploit the reverse of such mapping in order to automatically get a realization of the virtual blocks. Obviously, each available service places constraints on how the virtual blocks can be used, and the composition must be compatible with such constraints in order to actually exist.

Observe that in the Roman Model $[5,7]$, the available services are required to be deterministic, so as to capture their full controllability, in the sense that given a state and an action the result of the action on the service is a unique state. In other words, through actions the client can fully control the state transitions of the available services. Extensions of the Roman Model to nondeterministic, i.e., not fully controllable, available services have also been studied $[4,12]$ but are not subject of current paper. Also, the target service is required to be deterministic but for a different reason: to capture the full specification of the required composed target behavior. Indeed, nondeterminism at the level of target would correspond to a loose specification of the target itself, that is, instead of specifying a single target service to realize, a set of equally acceptable target services are specified (through nondeterminism), leaving the choice of which to realize to the composition system. Loose specifications of the target service have been studied in [6], and will not be considered further in this paper. It is worth mentioning that the Roman Model has been adapted and extended in several other directions. For example, in [14] look-head on the actions selected by the client of the target services are considered; in [15] composition services so as to realize batch sequences of actions is studied for 
various extensions of Roman Model; in [4] a framework based on the Roman Model, but that deals with data and explicit message exchange, is considered; finally, in [30] extensions of the Roman Model are investigated in the case a solution based on an orchestrator (see later) that mediates among the services is infeasible. Here as mentioned above here we do not deal with any of the above extensions, and concentrate on the basic proposal of $[5,7]$.

The main composition synthesis technique developed on the Roman Model is based on a reduction to satisfiability of a Propositional Dynamic Logic [19] formula. Such a reduction is polynomial and this gives an EXPTIME-upper-bound on the problem $[5,7]$. EXPTIME-hardness of the problem was recently shown by Muscholl and Walukiewicz [27].

In this paper we look again at such form of composition, but from a very different perspective, building on the following observation: a composition exists if and only if a simulation relation [26] exists from the target to the (nondeterministic) transition system formed by the asynchronous product of the available service transition systems. This observation was made several times by the authors of the paper in workshop and tutorials $[11,8,10]$, and it was also informally discussed in Daniela Berardi's $\mathrm{PhD}$ thesis [3], even if not fully worked out in a publication yet. The connection with simulation was also independently observed in [17], and although simulation is not explicitly mentioned, it is also related to the formal treatment of the extensions of the Roman model proposed in [14].

Once this observation is acquired we can develop a new technique for synthesizing composition, based on computing the maximal simulation and verifying whether the initial states of the target transition system and the asynchronous product of available transition systems are in the simulation. Such a computation is polynomial in the size of the target transition system and polynomial in the size of the asynchronous product of the available transition systems. As a result, the new technique is again in EXPTIME in the size of the available transition systems.

Besides these basic results, we show that synthesizing composition using simulation has a very interesting property: the maximal simulation contains enough information to allow for extracting every possible composition, through a suitable choice function. This property opens the possibility of devising composition in a "just-in-time" fashion: we compute the maximal simulation a priori then, equipped with such a simulation, we start executing the composition, choosing the next step in the composition according to criteria that can depend on information that is available only at run-time (actual availability of services, network communication problems or cost, etc.). Indeed, it suffices that the next step chosen for execution leads to service states that remain within the simulation relation. All in all, we believe that the synthesis technique proposed here provides the formal basis for building compositions that are reactive, i.e., able to deal with events that may occur at run-time.

The rest of the paper is organized as follows. First, in Section 2 and 3 we recall the notions of services and composition originally presented in $[5,7]$. In Section 4 we show the relationship between this kind of composition and simulation. 
In particular, we show how simulation can be used to check for the existence of composition in an optimal way from the computational complexity point of view. Also, at the end of the section, we make some remarks on the significance of this result in the context of simulation, where it closes a long standing open problem. In Section 5, we investigate the possibility of using simulation for actually synthesizing compositions, and we show how it can be used as a sort of precomputation that allows for generating composition in a "just-in-time" fashion at run-time. Finally, Section 6, concludes the paper.

\section{Services as Transition Systems}

In this section, we present the basic framework of our approach, starting from the description of services as finite transition systems (TSs). Besides these, further notions, which indeed characterize our approach, are introduced in order to formalize the intuitions exploited in the synthesis technique. The following paragraphs provide a detailed description of each notion, along with the ideas behind them.

Services Intuitively, a service is a software artifact characterized by its behavior, that is, the potential evolutions resulting from its interaction with some external system, such as a client service. A service is a program intended to interact with a client, whose interactions are expected to be conformant with a given behavior. More precisely, at each step, (i) the service presents the client a choice of available actions, according to its current state, (ii) the client instructs the service to execute one of them, (iii) the service executes it, moves to successor state and goes back to (i). Client-service interactions can be stopped, but does not have to, whenever the service is in a "final" state. Indeed, services that never terminate, thus offering a continuous interaction, are quite conceivable [7]. Also notice that, a service in general could start in an initial state that is not final, i.e., once started it needs to perform some action to get to a final state and terminate legally.

Since our technique aims at combining services in order to produce a desired behavior, a formal description of the above service behavior is needed. In this paper, a service (behavior) is represented by a finite deterministic transition system $T S=\left\langle A, S, s^{0}, \delta, F\right\rangle$, where:

- $A$ is the finite alphabet of actions;

- $S$ is the finite set of states;

- $s^{0}$ is the initial state;

- $\delta$ is the transition function (where $\delta(s, a)=s^{\prime}$ is represented by: $s \rightarrow_{a} s^{\prime}$ );

- $F$ is the set of final states.

Roughly speaking, a transition system looks like a state machine able to execute, according to the state it is in, actions taken from a shared alphabet $A$. However, its semantics is profoundly different. Consider the two transition systems in Figure 1. 

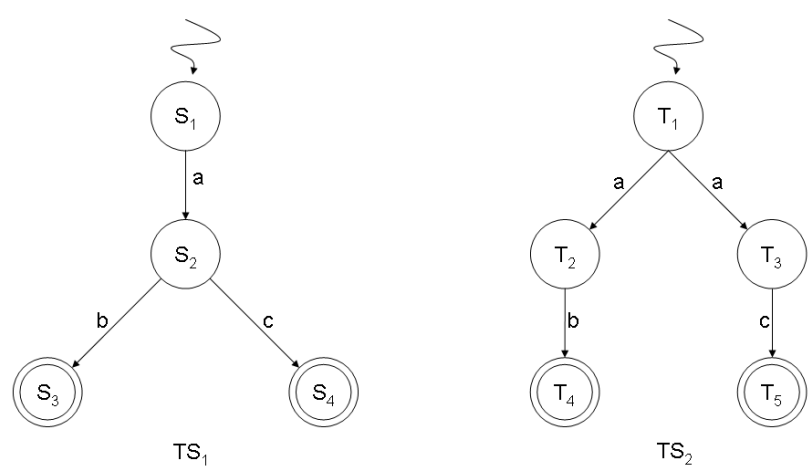

Figure 1: Two different transition systems

If they were finite automata they would be equivalent, since both represent the language $\{a b, a c\}$. But as transition systems they are indeed different:

- $T S_{1}$ is deterministic and models the case in which after action a one can perform both $b$ and $c$;

- $T S_{2}$ is nondeterministic and models the case in which after action $a$ one is allowed to perform either $b$ or $c$ depending on the nondeterministic choice of the transition for $a$.

In other words, the nondeterminism of finite state machines and language theory is angelic, and as a result nondeterminism becomes just a more compact way of representing the set of accepted action sequences. Instead, transition systems nondeterminism is devilish, i.e., the client can ask to perform an action but the actual transition is chosen (in a devilish way) by the transition system, that is, the service. Here, we follow the original proposal of the Roman Model and focus our attention on deterministic transition systems only.

Available services These are the services that correspond to existing programs, and are the only services directly available to the client. We remark that available services cannot be modified: they are defined once for all and evolve according to their behavior. The only way their evolution can be driven is by executing proper legal sequences of actions. In general, we deal with many -i.e., a community, see below- available services $\mathcal{S}_{i}(i=1, \ldots, n)$, each of them modeled as a transition system $T S_{i}=\left\langle A_{i}, S_{i}, s_{i}^{0}, \delta, F_{i}\right\rangle$.

Community A finite set of available services $\mathcal{C}=\left\{\mathcal{S}_{1}, \ldots, \mathcal{S}_{n}\right\}$ forms a community. The available services of a community share the same set of actions $A$-which 
is, indeed, the result of joining the action alphabets of all available services- even if some services might be not able to perform all actions in $A$.

Each community can be associated to a TS, which formalizes its "global" behavior: the result of combining in all possible ways the behaviors of its available services. Formally, such community transition system $\left(T S_{\mathcal{C}}\right.$, for community $\left.\mathcal{C}\right)$ is the asynchronous product of its available services. In details, let $T S_{1}, \ldots, T S_{n}$ be the TSs associated to the available services of $\mathcal{C}$, where $T S_{i}=\left\langle A, S_{i}, s_{i}^{0}, \delta_{i}, F_{i}\right\rangle$ $(i=1, \ldots, n)$, the community transition system $T S_{\mathcal{C}}=\left\langle A, S_{\mathcal{C}}, s_{\mathcal{C}}^{0}, \delta_{\mathcal{C}}, F_{\mathcal{C}}\right\rangle$ is defined as follows:

- $S_{\mathcal{C}}=S_{1} \times \ldots \times S_{n}$

- $s_{\mathcal{C}}^{0}=\left\langle s_{1}^{0}, \ldots, s_{n}^{0}\right\rangle$;

- $F_{\mathcal{C}}=F_{1} \times \ldots \times F_{n}$

- $\delta_{\mathcal{C}} \subseteq S_{\mathcal{C}} \times A \times S_{\mathcal{C}}$, where $\left(s_{1} \times \ldots \times s_{n}\right) \rightarrow_{a}\left(s_{1}^{\prime} \times \ldots \times s_{n}^{\prime}\right)$ iff:

- $\exists i$ s.t. $s_{i} \rightarrow_{a} s_{i}^{\prime}$

$-\forall j \neq i s_{j}^{\prime}=s_{j}$.

In general, despite the determinism of available services, $T S_{\mathcal{C}}$ may be non deterministic. Note that $T S_{\mathcal{C}}$ can execute a transition if and only if there exists one service among $T S_{1}, \ldots, T S_{n}$ that can do it and, consequently, can move to next state according to the transition performed by such service.

Target service Our goal is to synthesize, given a services community, a new service that realizes a desired behavior. Such a service is called target service and, again, is represented by a transition system $T S_{t}=\left\langle A_{t}, S_{t}, s_{t}^{0}, \delta_{t}, F_{t}\right\rangle$.

Notably, the target service is not one of the available services of the community, in general. Hence, it must be realized by exploiting fragments of the available service behaviors (computations), since these are the only services that correspond to existing programs in the system.

The following example makes actual the notions just introduced.

Example 1 [A multi-lingual community] Consider the services community depicted in Fig. 2, where available services provide several translation functionalities. Available services 2(a) and 2(b) provide, respectively, French-to-Italian and German-to-Italian translation services. Think of them as web services providing a page where the user first fills a form with some text and then can ask for its Italian translation. According to their TSs, translations can be asked for only after the form is filled out.

Similarly, available service 2(c) provides French-to-Italian translation functionalities, besides allowing for some further operations - such as finding synonymswhen German text is introduced (actually, the "sub-behavior" associated to such operations has been compacted in a single state, $\mathrm{S} 1$, since its details are not relevant for our purposes). Differently from previous services, Italian translation can 


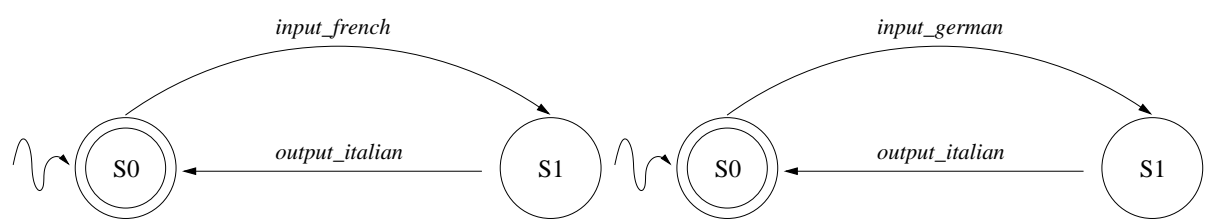

(a)

(b)

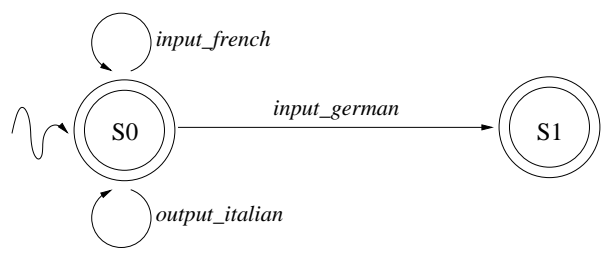

(c)

Figure 2: Example 1: Available services for a multi-lingual community

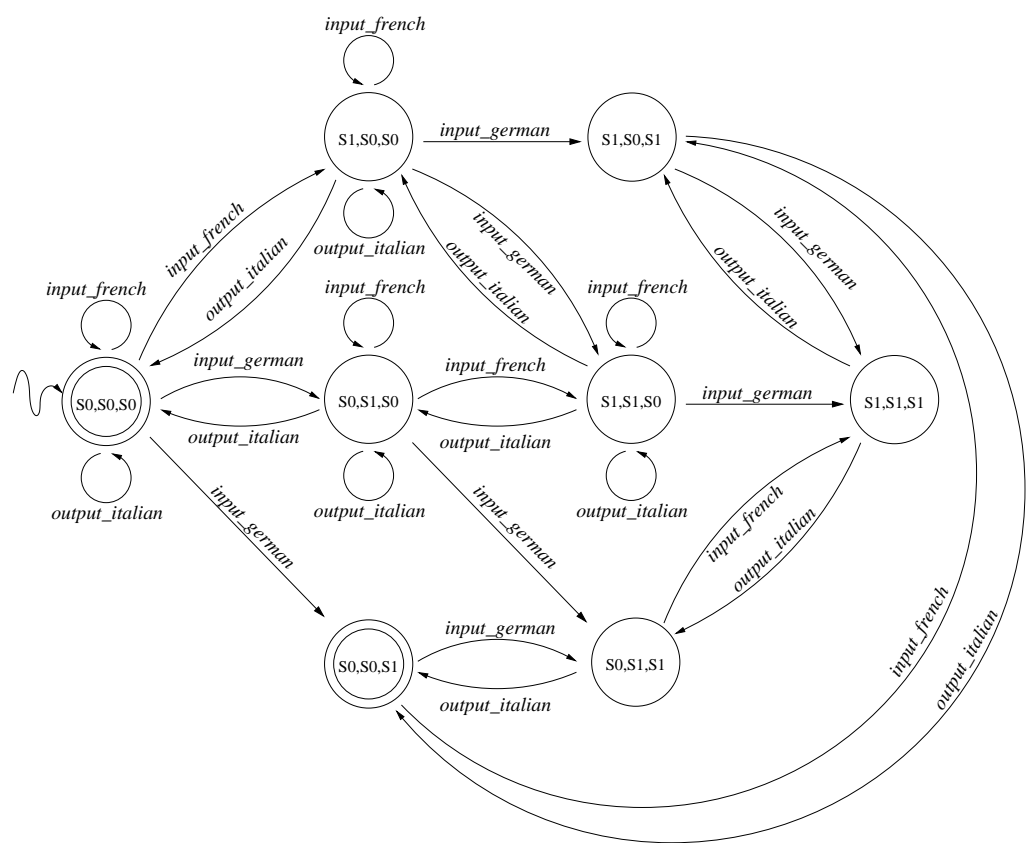

Figure 3: Example 1: community transition system 


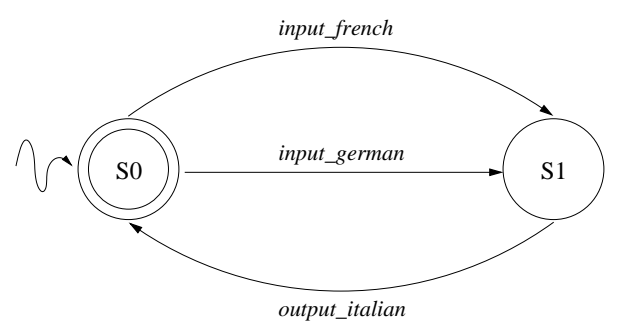

Figure 4: Example 1: target service

be performed even if no text is explicitly introduced, as shown by the looping edge on state S0, labeled by action output_italian -imagine that a buffer, initially filled out with some default text, is used to record the last translated input.

We will refer to TSs associated to services 2(a), 2(b) and 2(c) by means of subscripts $a, b$ and $c$, respectively. For instance, TS associated to service $2(\mathrm{a})$ is referred to as $T S_{a}=\left\langle A_{a}, S_{a}, s_{a}^{0}, \delta_{a}, F_{a}\right\rangle$. The community TS is represented by $T S_{\mathcal{C}}=\left\langle A_{\mathcal{C}}, S_{\mathcal{C}}, s_{\mathcal{C}}^{0}, \delta_{\mathcal{C}}, F_{\mathcal{C}}\right\rangle$

Finally, Fig. 3 shows the community transition system, which describes the behavior of the community seen as a whole system, where actions are performed by exactly one available service at a time. State labels are triples $\left\langle s_{a}, s_{b}, s_{c}\right\rangle \in S_{a} \times S_{b} \times S_{c}$ representing the state of each service after actions execution. Note that the community TS is non-deterministic.

Given such community, we are interested in synthesizing or, better said, composing, the target service depicted in Fig. 4, which allows for translating either French or German text to Italian.

\section{Service Composition}

Intuitively, the service composition problem can be stated as follows:

Given a target service and a community, synthesize a composition, i.e., a suitable function that delegates actions, requested by the client to the target service, to the available services in the community (which are the only services actually corresponding to existing programs).

As already discussed, both available and target services are represented by transition systems over a common actions alphabet $A$. Recall that (i) before any interaction takes place, each available service is in its initial state and (ii) a service can be left only if it is in a final state. Basically, composing a target service amounts to mimicking the desired (target) behavior by properly instructing, for each action chosen by the client (coherently evolving with the target service) a particular available service for performing the requested action. Of course, each time a service is to be selected for executing some action, the choice is constrained by the current state, as the result of the actions performed so far, of each available service (recall that a service evolves each time it interacts with the client). In addition, it obviously 
depends from future actions that, coherently with the target behavior, can be later requested by the client.

In order to make such intuition precise we first introduce the notion of execution tree.

Execution trees TSs provide a compact description of service abilities, but take into account no issue concerning their actual evolution. If, on one hand, TSs describe which actions a service can execute and how its state changes, on the other hand, they do not keep track of how states are reached. As a matter of fact, in general, a given state may result from the execution of different action sequences, and the state itself holds no information about which of them has been actually executed. Since this aspect is crucial for our purposes, a formal definition is required.

The actual evolution of a service can be described by an execution tree. Intuitively, it is a structure obtained by "unfolding" the TS associated to the service. More formally, given a service $\mathcal{S}$ and its associated transition system $T S=\left\langle A, S, s^{0}, \delta, F\right\rangle$, an execution tree for $\mathcal{S}$ is a pair $\langle\mathcal{T}$, final $\rangle$, where $\mathcal{T}$ is a tree over $A$ (i.e., a prefixed closed set of string over $A$ ) and final is a boolean function over nodes of $\mathcal{T}$. Both $\mathcal{T}$ and final are inductively defined by making use of an auxiliary function $m_{T S}: \mathcal{T} \rightarrow S$, as follows:

- $\varepsilon \in \mathcal{T}$, and $m_{T S}(\varepsilon)=s_{0}$, i.e., $m_{T S}$ associates the root $\varepsilon$ of $\mathcal{T}$ to the initial state $s_{0}$ of $T S$;

- let $x \in \mathcal{T}$, and $m_{T S}(x)=s$ where $s \in S$ : if $s \rightarrow_{a} s^{\prime}$ then $x \cdot a \in \mathcal{T}$, i.e., $x$ has an $a$ successor, and $m_{T S}(x \cdot a)=s^{\prime}$;

- $\operatorname{final}(x)=$ true iff $m_{T S}(x) \in F$.

Observe that each node of $\mathcal{T}$ is a sequence of actions $x=a_{1} \cdots \cdots a_{k}$ allowed in $T S$, starting from the initial state. Such sequences are called histories for TS. Then, each node $x=a_{1} \cdots \cdots a_{k}$ of execution tree $\mathcal{T}$ represents a history for $T S$. Given a history $a_{1} \cdots a_{k}$, we do know the state of $T S$ after its execution, starting from the initial state, namely $m_{T S}\left(a_{1} \cdots a_{k}\right)$. Also, notice that, given a node $x=a_{1} \cdots \cdots a_{k}$ of $\mathcal{T}$, its successor nodes, namely $x \cdot a_{k+1}^{1}, \ldots, x \cdot a_{k+1}^{\ell}$, tell us which actions, namely $a_{k+1}^{1}, \ldots, a_{k+1}^{\ell}$, are allowed in the current state of $T S$, that is, the state reached from the initial state through history $a_{1} \cdots \cdots a_{k}$. Furthermore, function final(.) tells us whether through the history $x, T S$ has reached a final state, i.e., whether $\operatorname{final}(x)=$ true.

Example 2 Fig. 5 depicts the execution tree generated by system 2(c). Note that, coherently with its transition system: (i) every state is final, (ii) action input_german always leads to a sink node where no further action can be performed and (iii) whenever execution is in a state where either input_french or output_italian can be performed, any arbitrary sequence of such actions is allowed. Construction of execution trees for systems 2(a) and 2(b) is straightforward. 


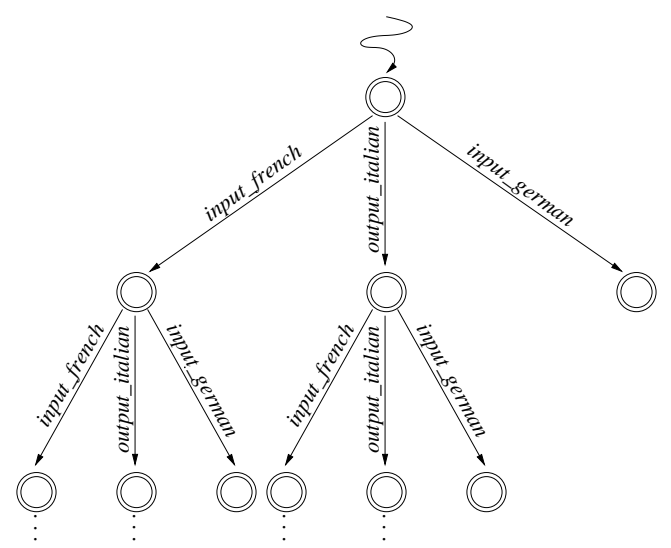

Figure 5: Example 1: execution tree of service (c)

Composition With the notion of TS execution tree in place, we can formally define service composition. The crux notion is that of composition labeling which formalizes the idea of assigning actions to services.

Definition 1 (Composition labeling) Let $\mathcal{C}=\left\{\mathcal{S}_{1}, \ldots, \mathcal{S}_{n}\right\}$ be a community of available services, $\mathcal{S}_{t}$ be the target service and $\mathcal{T}_{i} \mathcal{S}=\left\langle\mathcal{T}_{i}\right.$, final $\left.{ }_{i}\right\rangle$ be the execution tree for $\mathcal{S}_{i}(i=1, \ldots, n, t)$. A composition labeling of $\mathcal{T}_{t}^{\mathcal{S}}$ wrt $\mathcal{T}_{1}^{\mathcal{S}}, \ldots, \mathcal{T}_{n}^{\mathcal{S}}$ is a function $\mathrm{CLAB}: \mathcal{T}_{t}^{\mathcal{S}} \rightarrow \mathcal{T}_{1}^{\mathcal{S}} \times \cdots \times \mathcal{T}_{n}^{\mathcal{S}}$ that satisfies the following conditions:

1. $\operatorname{CLAB}(\varepsilon)=\langle\varepsilon, \ldots, \varepsilon\rangle$;

2. for every node $x \in \mathcal{T}_{t}$, let $\operatorname{CLAB}(x)=\left\langle x_{1}, \ldots, x_{n}\right\rangle$; then, for all $a \in A$ such that $x \cdot a \in \mathcal{T}_{t}, \operatorname{CLAB}(x \cdot a)=\left\langle y_{1}, \ldots, y_{n}\right\rangle$, where $y_{i}=x_{i} \cdot a$ for exactly one $i \in[1, \ldots, n]$ (if service $\mathcal{S}_{i}$ performs interaction a) and $y_{j}=x_{j}$ otherwise.

3. for every node $x \in \mathcal{T}_{t}$, if final $(x)=$ true and $\operatorname{CLAB}(x)=\left\langle x_{1}, \ldots, x_{n}\right\rangle$, then final $_{i}\left(x_{i}\right)=$ true for $i=1, \ldots, n$.

Intuitively, CLAB labels each node of the target service execution tree $\mathcal{T}_{t}^{\mathcal{S}}$ with a tuple $\left\langle x_{1}, \ldots, x_{n}\right\rangle$, where the generic component $x_{i}(i=1, \ldots, n)$ denotes the current node of execution tree $\mathcal{T}_{i}^{\mathcal{S}}$, i.e., the history of actions executed so far, starting from the initial state, by $i$-th available service. Requirement (1) states that all services start from their respective initial state; requirement (2) constrains each action of the target service to be executed by exactly one available service (in its current state, which results from its history so far), while the other services remain still; finally, requirement (3) allows for leaving the target service only if all available services are in a final configuration. Summing up, CLAB relates, in a step-by-step fashion, the evolution of the target service to the evolution of available services, by suitably delegating, in a step-by-step fashion actions requested to the target services to one of the available services.

Given a composition labeling CLAB, one can orchestrate the $n$ available services to mimic the target service $\mathcal{S}_{t}$ by stepping each available service according to what 
is specified by CLAB itself. Thus, service composition can be formally defined as follows:

Definition 2 (Service composition) A composition of the services in the community $\mathcal{C}=\left\{\mathcal{S}_{1}, \ldots, \mathcal{S}_{n}\right\}$ realizing the target service $\mathcal{S}_{t}$ is a function COMP : $\mathcal{T}_{t}^{\mathcal{S}} \rightarrow$ $\{1, \ldots, n\} \cup \perp$ such that

- $\operatorname{COMP}(\varepsilon)=\perp$

- $\operatorname{COMP}(x \cdot a)=i$, where $\operatorname{CLAB}(x)=\left\langle y_{1}, \ldots, y_{i}, \ldots, y_{n}\right\rangle$ and $\operatorname{CLAB}(x \cdot a)=$ $\left\langle y_{1}, \ldots, y_{i} \cdot a, \ldots, y_{n}\right\rangle$, i.e., $\operatorname{CLAB}(x)$ and $\operatorname{CLAB}(x \cdot a)$ are identical except for the $i$-th component that from $y_{i}$ in $\operatorname{CLAB}(x)$ becomes $y_{i} \cdot a$ in $\operatorname{CLAB}(x \cdot a)$.

Observe that, by definition, given a composition labeling CLAB we get the corresponding composition COMP. The vice-versa is also true: given a composition COMP, it is immediate to get the corresponding composition labeling CLAB, as follows:

- $\operatorname{CLAB}(\varepsilon)=\langle\varepsilon, \ldots, \varepsilon\rangle$;

- for every node $x \in \mathcal{T}_{t}$, let $\operatorname{CLAB}(x)=\left\langle x_{1}, \ldots, x_{n}\right\rangle$; then for all $a \in A$ such that $x \cdot a \in \mathcal{T}_{t} \operatorname{CLAB}(x \cdot a)=\left\langle y_{1}, \ldots, y_{n}\right\rangle$, where $y_{i}=x_{i} \cdot a$ if $\operatorname{COMP}(x \cdot a)=i$, and $y_{j}=x_{j}$ otherwise.

Computational complexity characterization Composition, as defined above, has already been studied in $[5,7]$. In particular, the computational complexity characterization of the service composition problem is known. The upper bound was established in [5]:

Theorem 1 ([5]) Checking the existence of a composition of the services in a community $\mathcal{C}=\left\langle\mathcal{S}_{1}, \ldots, \mathcal{S}_{n}\right\rangle$ that realizes a target service $\mathcal{S}_{t}$ can be done in EXPTIME. A matching lower bound was recently proved by Muscholl and Walukiewicz:

Theorem 2 ([27]) Checking the existence of a composition of the services in a community $\mathcal{C}=\left\langle\mathcal{S}_{1}, \ldots, \mathcal{S}_{n}\right\rangle$ that realizes a target service $\mathcal{S}_{t}$ is EXPTIME-hard.

In other words, checking the existence of a composition is an EXPTIME-complete problem.

Notably, in $[5,7]$ an actual synthesis technique for computing the composition is presented. Such a technique is based on a polynomial reduction to satisfiability in Propositional Dynamic Logic [19]. Here, however, we do not rely on such a technique. Instead, we develop a new composition synthesis technique based on the notion of simulation.

\section{Composition and Simulation}

Now, we illustrate the basic result of this paper: we show that checking for the existence of a service composition can be done by checking for the existence of a simulation relation between the target and the community TSs. We start by defining the notion of simulation relation [26] in our context.

Definition 3 (Simulation relation) Given two transition systems $T S_{t}$ and $T S_{\mathcal{C}}$, a simulation relation of $T S_{t}$ by $T S_{\mathcal{C}}$ is a relation $R \subseteq S_{t} \times S_{\mathcal{C}}$, such that: 


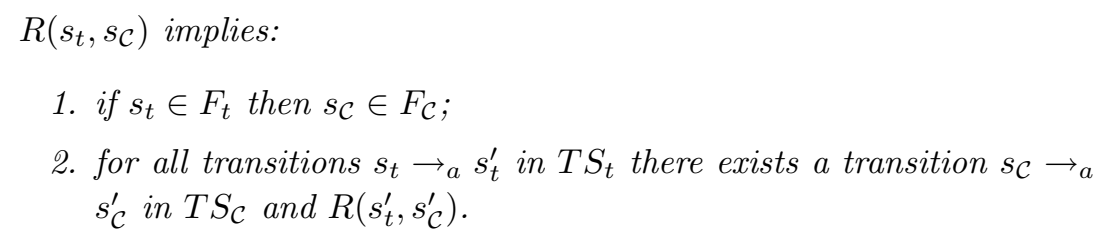

The definition says that state $s_{t}$ of $T S_{t}$ is in a simulation relation $R$ with $s_{\mathcal{C}}$ of $T S_{\mathcal{C}}$ if: (i) if $s_{t}$ is final then also $s_{\mathcal{C}}$ is final; (ii) for every action $a$ and state $s_{t}^{\prime}$, if $s_{t}$ can make a transition to $s_{t}^{\prime}$ with action $a$, then also $s_{\mathcal{C}}$ can make a transition to some $s_{\mathcal{C}}^{\prime}$ with action $a$, in such a way that $s_{t}^{\prime}$ is still in the same simulation relation $R$ with $s_{C}^{\prime}$. Observe the coinductive nature of such a definition: indeed it is cyclic but with no base case.

Definition 4 Let $T S_{t}$ be the transition system representing the target service, and $T S_{\mathcal{C}}$ be the community transition system. A state $s_{t} \in S_{t}$ is simulated by a state $s_{\mathcal{C}} \in S_{\mathcal{C}}$ (or $s_{\mathcal{C}}$ simulates $\left.s_{t}\right)$, denoted $s_{t} \preceq s_{\mathcal{C}}$, iff there exists a simulation $R$ of $T S_{t}$ by $T S_{\mathcal{C}}$ s.t $R\left(s_{t}, s_{\mathcal{C}}\right)$.

Observe that the relation $\preceq$ is itself a simulation relation. In fact, it is the largest simulation relation since, by the definition above, all simulation relations are contained in $\preceq$.

Definition $5 T S_{t}$ is simulated by $T S_{\mathcal{C}}$ (or $T S_{\mathcal{C}}$ simulates $T S_{t}$ ) iff $s_{t}^{0} \preceq s_{\mathcal{C}}^{0}$, where $s_{t}^{0}$ and $s_{\mathcal{C}}^{0}$ are the initial states of the target and the community TSs, respectively.

Example 3 [Example 1, continued] Consider the target (Fig. 4) and the community (Fig. 3) services of Example 1. In Figure 6, a simulation of the former service by the asynchronous product of the latter ones is given. Dashed lines associate each state of the target TS to those states of the community TS it is simulated by. For instance, state $\langle\mathrm{S} 1, \mathrm{~S} 0, \mathrm{~S} 0\rangle$ of $T S_{\mathcal{C}}$ simulates state $\mathrm{S} 1$ of $T S_{t}$ as well as state $\mathrm{S} 0$ is simulated by both $\langle\mathrm{S} 0, \mathrm{~S} 0, \mathrm{~S} 0\rangle$ and $\langle\mathrm{S} 0, \mathrm{~S} 0, \mathrm{~S} 1\rangle$. Note that, in general, there may exist several simulations. The one shown in Figure 6 represents, in fact, the largest one, i.e., the relation $\preceq$.

Theorem 3 below shows how checking for the existence of a service composition can be reduced to checking whether the target transition system is simulated by the community transition system. In order to prove such result, we need to introduce two preliminary lemmas.

Lemma 1 Let $\mathcal{C}=\left\{\mathcal{S}_{1}, \ldots, \mathcal{S}_{n}\right\}$ be a community, $\mathcal{S}_{t}$ a target service, and CLAB a composition labeling of $\mathcal{T}_{t}^{\mathcal{S}}$ wrt $\mathcal{T}_{1}^{\mathcal{S}}, \ldots, \mathcal{T}_{n}^{\mathcal{S}}$. Then the relation $R \subseteq$ $S_{t} \times S_{\mathcal{C}}$ defined as $R=\left\{\left\langle s_{t}, s_{c}\right\rangle \mid \exists x, x_{1}, \ldots, x_{n}: m_{T S_{t}}(x)=s_{t}, \operatorname{CLAB}(x)=\right.$ $\left.\left\langle x_{1}, \ldots, x_{n}\right\rangle,\left\langle m_{T S_{1}}\left(x_{1}\right), \ldots, m_{T S_{n}}\left(x_{n}\right)\right\rangle=s_{c}\right\}$ is a simulation relation of $T S_{t}$ by $T S_{\mathcal{C}}$ such that $R\left(s_{t}^{0}, s_{\mathcal{C}}^{0}\right)$.

Proof. The following arguments prove that $R$ is a simulation:

- Since $\operatorname{CLAB}(\varepsilon)=\langle\varepsilon, \ldots, \varepsilon\rangle$ by definition of CLAB, then $R\left(s_{t}^{0}, s_{\mathcal{C}}^{0}\right)$ holds by definition of $R$. 


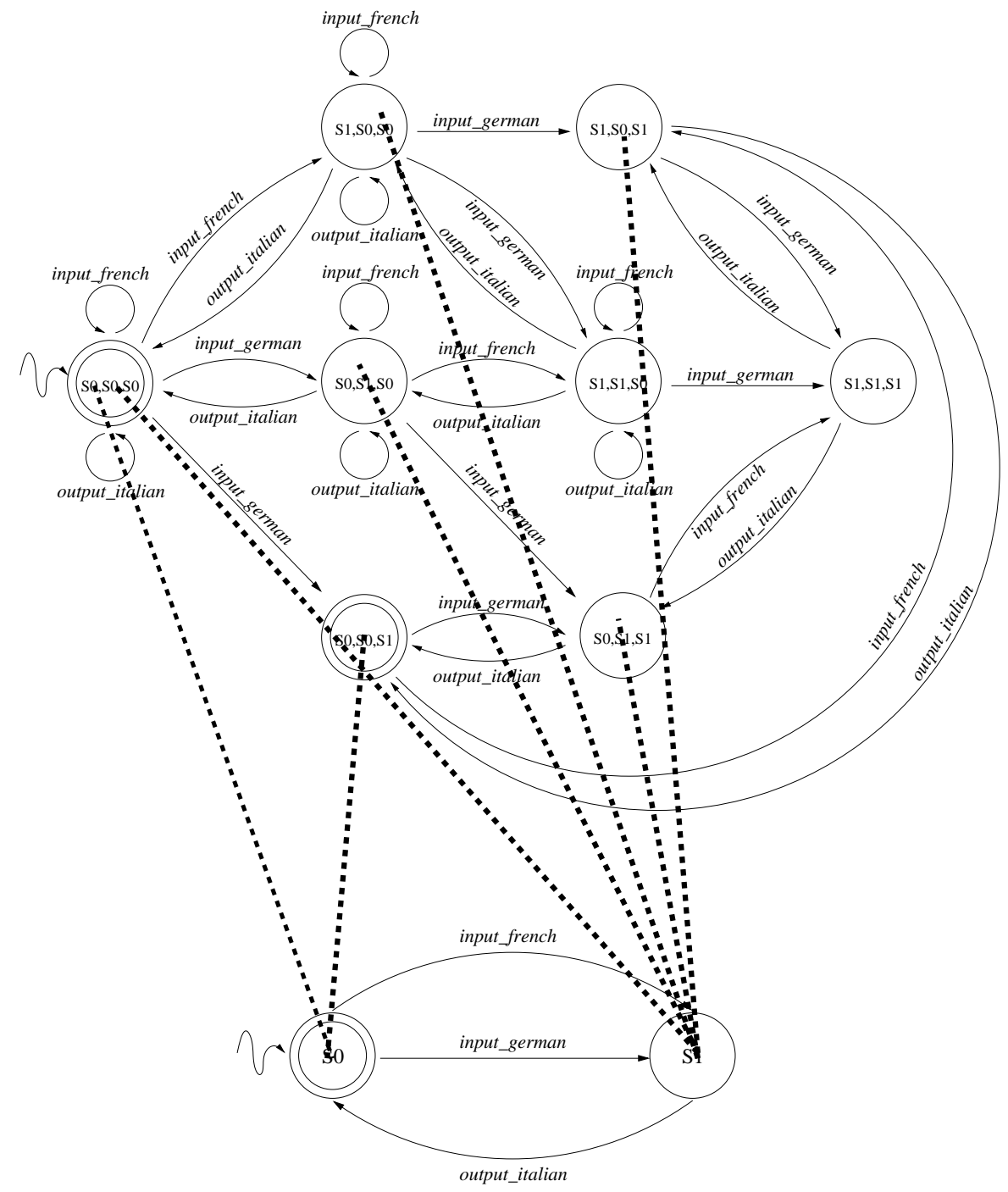

Figure 6: Example 3: A simulation relation for Example 1 
- Consider a final node $x \in \mathcal{T}_{t}$ associated to a final state $s_{t} \in F_{t} \subseteq S_{t}$ by $m_{T S_{t}}(x)=s_{t}$. Recall that $s_{t}$ is final iff $x$ does. By definition of $R, x$ is associated, by CLAB, to a tuple $\left\langle x_{1}, \ldots, x_{n}\right\rangle$ such that $\left\langle m_{T S_{1}}\left(x_{1}\right), \ldots, m_{T S_{n}}\left(x_{n}\right)\right\rangle=$ $s_{\mathcal{C}}$. By definition of CLAB, being $x$ final, also $x_{1}, \ldots, x_{n}$ do. By definition of $m_{T S_{i}}$ each $s_{i}$ is final, therefore $s_{\mathcal{C}}$ is final.

- Let $m_{T S_{t}}(x)=s_{t}, x^{\prime}=x \cdot a, s_{t} \rightarrow_{a} s_{t}^{\prime}$ and $m_{T S_{t}}\left(x^{\prime}\right)=s_{t}^{\prime}$. By definition of $R$, $\operatorname{CLAB}(x)=\left\langle x_{1}, \ldots, x_{n}\right\rangle$ and $\left\langle m_{T S_{1}}\left(x_{1}\right), \ldots, m_{T S_{n}}\left(x_{n}\right)\right\rangle=s_{\mathcal{C}}$. By definition of $\operatorname{CLAB}, \operatorname{CLAB}(x \cdot a)=\left\langle x_{1}^{\prime}, \ldots, x_{n}^{\prime}\right\rangle$, where for one $i \in[1, \ldots, n]$, we get $x_{i}^{\prime}=x_{i} \cdot a$, and for all other $j \in[1, \ldots, n]$ with $j \neq i$, we get $x_{j}^{\prime}=x_{j}$. Finally, by definition of $m_{T S_{i}}, m_{T S_{i}}\left(x_{i}^{\prime}\right)=s_{i}^{\prime}$ iff $s_{i} \rightarrow_{a} s_{i}^{\prime}$. Hence, $s_{\mathcal{C}} \rightarrow_{a} s_{\mathcal{C}}^{\prime}$ and, consequently, $R\left(s_{t}^{\prime}, s_{\mathcal{C}}^{\prime}\right)$ holds.

The lemma above constructively states that, given a target service $\mathcal{S}_{t}$ and a community $\mathcal{C}$, for every composition labeling of the execution tree associated to $\mathcal{S}_{t}$ by the execution trees of community services, it is always possible to build a relation $R$ which is a simulation of $T S_{t}$ by $T S_{\mathcal{C}}$.

Lemma 2 Let $\mathcal{C}=\left\{\mathcal{S}_{1}, \ldots, \mathcal{S}_{n}\right\}$ be a community, $\mathcal{S}_{t}$ a target service, and $R$ a simulation relation of $T S_{t}$ by $T S_{\mathcal{C}}$ such that $R\left(s_{t}^{0}, s_{\mathcal{C}}^{0}\right)$. Then, there exists a composition labeling CLAB of $\mathcal{T}_{t}^{\mathcal{S}}$ wrt $\mathcal{T}_{1}^{\mathcal{S}}, \ldots, \mathcal{T}_{n}^{\mathcal{S}}$.

Proof. Let $R$ be a simulation of $T S_{t}$ by $T S_{\mathcal{C}}$ such that $R\left(s_{t}^{0}, s_{\mathcal{C}}^{0}\right)$ where $s_{\mathcal{C}}^{0}=$ $\left\langle s_{1}^{0}, \ldots, s_{n}^{0}\right\rangle$. From $R$ we can build a labeling function CLAB : $\mathcal{T}_{t}^{\mathcal{S}} \rightarrow \mathcal{T}_{1}^{\mathcal{S}} \times \cdots \times \mathcal{T}_{n}^{\mathcal{S}}$, by induction on the level of nodes in $\mathcal{T}_{t}$, which shows that a composition does exist. Recall that (i) $R$ associates each state of $T S_{t}$ to a tuple of states from $T S_{1} \times \ldots \times T S_{n}$ (that is, the set of community TS states) and (ii) a mapping $m_{T S_{i}}$ associates each node of $\mathcal{T}_{i}$ to a corresponding state of $T S_{i}(i=1, \ldots, n, t)$. We proceed as follows:

- Base case.

$m_{T S_{t}}(\varepsilon)=s_{t}^{0}$, i.e. the root of $\mathcal{T}_{t}$ is labeled with the initial state of $T S_{t}$, and analogously for each $T S_{i}$. Since $R$ is a simulation, we have that $R\left(s_{t}^{0},\left\langle s_{1}^{0}, \ldots, s_{n}^{0}\right\rangle\right)$. Therefore, we define $\operatorname{CLAB}(\varepsilon)=\langle\varepsilon, \ldots, \varepsilon\rangle$.

- Inductive hypothesis.

Let $m_{T S_{t}}(x)=s_{t}$ and let $R\left(s_{t},\left\langle s_{1}, \ldots, s_{n}\right\rangle\right)$. Let $\operatorname{CLAB}(x)=\left\langle x_{1}, \ldots, x_{n}\right\rangle$, where $m_{T S_{i}}\left(x_{i}\right)=s_{i}$.

- Induction step.

Let $x^{\prime}=x \cdot a$ be a successor node of $x$. If such a node exists, there exists also a transition $s_{t} \rightarrow_{a} s_{t}^{\prime}$ such that $m_{T S_{t}}\left(x^{\prime}\right)=s_{t}^{\prime}$. Therefore, since $R\left(s_{t},\left\langle s_{1}, \ldots, s_{n}\right\rangle\right)$ holds by inductive hypothesis, then a tuple ${ }^{\mathrm{a}}\left\langle s_{1}^{\prime}, \ldots, s_{n}^{\prime}\right\rangle$ exists such that $R\left(s_{t}^{\prime},\left\langle s_{1}^{\prime}, \ldots, s_{n}^{\prime}\right\rangle\right)$. Such a tuple, by definition of $T S_{\mathcal{C}}$, must be such that for one $i \in[1, \ldots, n]$, we have $s_{i} \rightarrow_{a} s_{i}^{\prime}$ and for all other $j \in[1, \ldots, n]$ with $j \neq i$, we have that $s_{j}^{\prime}=s_{j}$. Hence, we can define $\operatorname{CLAB}\left(x^{\prime}\right)=\left\langle x_{1}^{\prime}, \ldots, x_{n}^{\prime}\right\rangle$, where $m_{T S_{t}}\left(x_{i}^{\prime}\right)=s_{i}^{\prime}$ and:

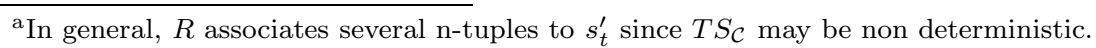




$$
\begin{aligned}
& - \text { if } s_{i}^{\prime}=s_{i} \text { then } x_{i}^{\prime}=x_{i} \\
& - \text { if } s_{i} \rightarrow_{a} s_{i}^{\prime} \text { then } x_{i}^{\prime}=x_{i} \cdot a
\end{aligned}
$$

Finally, recall that each $\mathcal{T}_{t}$ 's final node is associated, through $m_{T S_{t}}$, to exactly one $T S_{t}$ 's final state. Let $x$ be one of such nodes and let $m_{T S_{t}}(x)=s_{t}$. Since $R$ is a simulation, it relates $s_{t}$ to some tuple(s) $\left\langle s_{1}, \ldots, s_{n}\right\rangle$, where each component is a final state for the TS it refers to. Hence being $\operatorname{CLAB}(x)=\left\langle x_{1}, \ldots, x_{n}\right\rangle$, with $m_{T S_{i}}\left(x_{i}\right)=s_{i}$, we get that $x_{1}, \ldots, x_{n}$ are final. Concluding, we get that CLAB, defined as above, is indeed a composition labeling.

This lemma says that given a simulation $R$ of $T S_{t}$ by $T S_{\mathcal{C}}$, the whole set of composition labelings which realize the target service can be always defined. Note that such set is not a singleton since, in general, $R$ associates a $T S_{t}$ 's state to many (possibly one) $T S_{\mathcal{C}}$ 's states. Observe that also the proof of this lemma is constructive.

As a direct consequence of Lemmas 1 and 2, we get our theorem.

Theorem $3 A$ composition of the services in the community $\mathcal{C}=\left\{\mathcal{S}_{1}, \ldots, \mathcal{S}_{n}\right\}$ realizes the target service $\mathcal{S}_{t}$ if and only if $T S_{t}$ is simulated by $T S_{\mathcal{C}}$.

Proof. By definition of composition, it suffices to prove that there exists a composition labeling of $\mathcal{T}_{t}^{\mathcal{S}}$ wrt $\mathcal{T}_{1}^{\mathcal{S}}, \ldots, \mathcal{T}_{n}^{\mathcal{S}}$ if and only if $T S_{t}$ is simulated by $T S_{\mathcal{C}}$, which is a consequence of Lemma 1 for " $\Rightarrow$ " direction and Lemma 2 for " $\Leftarrow$ " direction.

Theorem 3 gives us a straightforward method to check for the existence of composition, namely:

- compute the maximal simulation relation $\preceq$ of $T S_{t}$ by $T S_{\mathcal{C}}$;

- check whether $\left\langle s_{t}^{0}, s_{\mathcal{C}}^{0}\right\rangle$ is in such a relation.

Observe that such a method is quite different from the one in [5] which was based on a polynomial reduction to satisfiability in Propositional Dynamic Logic [19].

From the computational point of view, we recall that checking for the existence of a simulation relation between two (states of two) transition systems can be done in polynomial time in the size of the transition systems - moreover well developed techniques exists for computing simulation, such as those in [20, 32, 13]. Since in our case the number of states of $T S_{\mathcal{C}}$ is exponential in the size (i.e., the number of states) of $T S_{1}, \ldots, T S_{n}$, we get that we can check for the existence of a composition using simulation in exponential time. Considering that the problem is EXPTIMEcomplete, we get the following result:

Theorem 4 Checking the existence of compositions via simulation is optimal with respect to worst-case complexity.

Notably, the EXPTIME-completeness of service composition, gives us the following result in the context of simulation, which closes a long standing open problem in the simulation literature: 
Theorem 5 Checking simulation from a single deterministic transition system to the asynchronous product of to $n$ deterministic transition systems is an EXPTIMEcomplete problem.

Proof. The membership to EXPTIME is a direct consequence of Theorem 1 and Theorem 4. As for the EXPTIME-hardness, if a lower complexity technique would exist for the simulation problem above it could be applied to service composition as well, leading to a lower complexity technique for service composition itself and thus contradicting the EXPTIME-hardness result of Theorem 2.

Indeed, in [31] the computational complexity characterization of checking simulation from the asynchronous product of $n$ concurrent deterministic transition systems to a single deterministic transition system was given, however the computational complexity characterization of checking simulation in the converse direction has remained open since. We close it here, by transferring EXPTIME-completeness result of service composition to simulation.

\section{Synthesizing Composition via Simulation}

Theorem 3 closely relates the notion of simulation relation to the one of service composition showing, ultimately, that finding a service composition corresponds to finding a simulation relation between two particular - the target and the communitytransition systems and vice-versa. However, no procedure is given for actually synthesizing an orchestrator that implements such a composition by properly assigning action executions to available services. In this section, we show that if a simulation relation exists, it can be used to synthesize an orchestrator. To this end, we refer to an abstract structure called orchestrator generator, or simply $O G$. Intuitively, the $O G$ is a program that returns, for each state reached by the community in realizing a target history, the set of available services capable of performing the (target-conformant) action the client requests next. $O G$ is directly obtained from the maximal simulation relation between the target and the community TSs.

Definition 6 (Orchestrator Generator, $\boldsymbol{O} \boldsymbol{G}$ ) Let $\mathcal{S}_{t}$ be a target service and $\mathcal{C}=\left\{\mathcal{S}_{1}, \ldots, S_{n}\right\}$ be a community of available services such that $T S_{t}$ is simulated by $T S_{\mathcal{C}}$. The orchestrator generator $(O G)$ for $T S_{t}$ and $T S_{\mathcal{C}}$ is a tuple $O G=\left\langle A,[1, \ldots, n], S_{r}, s_{r}^{0}, \omega_{r}, \delta_{r}, F_{r}\right\rangle$, where:

1. A is the finite set of community actions;

2. $[1, \ldots, n]$ is the set of available services indices;

3. $S_{r}=S_{t} \times S_{1} \times \ldots \times S_{n}$ is the set of $O G$ states;

4. $s_{r}^{0}=\left\langle s_{t}^{0}, s_{1}^{0}, \ldots, s_{n}^{0}\right\rangle$ is the $O G^{\prime} s$ initial state;

5. $F_{r}=\left\{\left(s_{t}, s_{1}, \ldots, s_{n}\right) \mid s_{i} \in F_{i}\right.$, for $\left.i=t, 1, \ldots, n\right\}$ is the set of $O G$ 's final states;

6. $\omega_{r}: S_{r} \times A \mapsto 2^{[1, \ldots, n]}$ is the service selection function: let $s_{r}=\left\langle s_{t}, s_{1}, \ldots, s_{n}\right\rangle \in S_{r}, \omega_{r}\left(s_{r}, a\right)$ is defined iff 
- $s_{t} \preceq\left\langle s_{1}, \ldots, s_{n}\right\rangle$ and

- there exists $s_{t}^{\prime}$ s.t $s_{t} \rightarrow_{a} s_{t}^{\prime}$;

in such case, $\omega_{r}\left(s_{r}, a\right)=\left\{k \mid \exists s_{k}^{\prime} . s_{k} \rightarrow_{a} s_{k}^{\prime} \wedge s_{t}^{\prime} \preceq\left\langle s_{1}, \ldots, s_{k}^{\prime}, \ldots, s_{n}\right\rangle\right\} ;$

7. $\delta_{r}: S_{r} \times A \times[1, \ldots, n] \rightarrow S_{r}$ is the transition function.

$\delta_{r}\left(s_{r}, a, k\right)$ is defined iff $k \in \omega_{r}\left(s_{r}, a\right)$, as follows:

$\delta_{r}\left(s_{r}, a, k\right)=s_{r}^{\prime}$, where $s_{r}^{\prime}=\left\langle s_{t}^{\prime}, s_{1}, \ldots, s_{k}^{\prime}, \ldots, s_{n}\right\rangle, s_{t} \rightarrow_{a} s_{t}^{\prime}$ and, $s_{k} \rightarrow_{a} s_{k}^{\prime}$.

Intuitively, $O G$ is a finite state machine that, given a (target-conformant) action $a$, outputs (function $\omega_{r}$ ) the set of services which can perform $a$ next according to the maximal simulation relation $\preceq$. For each choice of one of such services it progresses to the next state (function $\delta_{r}$ ).

Once we have $O G$, we get orchestrators by simply picking up, at each step, one among the services returned by $\omega_{r}$. Formally, we define a (generated) orchestrator as follows:

Definition 7 (Generated Orchestrator) Given an orchestrator generator $O G$ for $T S_{t}$ and $T S_{\mathcal{C}}$, defined as above, a generated orchestrator is a function $\mathrm{ORCH}$ : $\mathcal{T}_{t} \rightarrow[1, \ldots, n] \cup \perp$, inductively defined as follows:

- $\operatorname{ORCH}(\varepsilon)=\perp$;

- if $x \cdot a \in \mathcal{T}_{t}$, then $\operatorname{ORCH}(x \cdot a)=i \in \omega_{r}\left(\sigma_{r}^{\mathrm{ORCH}}(x), a\right)$, where: $\sigma_{r}^{\mathrm{ORCH}}: \mathcal{T}_{t} \rightarrow S_{r}$ is the mapping function between nodes of $\mathcal{T}_{t}$ and corresponding states of $S_{r}$, defined as follows:

$$
\begin{aligned}
& -\sigma_{r}^{\mathrm{ORCH}}(\varepsilon)=s_{r}^{0} \\
& - \text { if } x \cdot a \in \mathcal{T}_{t} \text { then } \sigma_{r}^{\mathrm{ORCH}}(x \cdot a)=\delta_{r}\left(\sigma_{r}^{\mathrm{ORCH}}(x), a, \operatorname{ORCH}(x \cdot a)\right)
\end{aligned}
$$

A generated orchestrator is, basically, a function which selects an available service for executing the action requested by the (target-conformant) client. In order to guarantee the selected service to be actually capable of executing the assigned action, orchestrator assignments must belong to the set defined by $\omega_{r}$, at each step. Note that such set depends on $\sigma_{r}^{\mathrm{ORCH}}$ which, in turns, depends on ORCH itself. $\sigma_{r}^{\mathrm{ORCH}}$ maps each node of target service execution tree, that is a target history, into the state reached by the community TS when such history is actually executed. Since, also, ORCH depends on $\sigma_{r}^{\mathrm{ORCH}}$, both functions are defined by mutual induction, through $\omega_{r}$. Note how such induction is well-founded, since $i$-th step value of oRCH depends, through $\omega_{r}$, on $(i-1)$-th step value of $\sigma_{r}^{\mathrm{ORCH}}$.

Example 4 [Example 3, continued] As an $O G$ instance, consider Figure 7, where a graphical representation, i.e., a graph, of $O G=\left\langle A,[1, \ldots, n], S_{r}, s_{r}^{0}, \omega_{r}, \delta_{r}, F_{r}\right\rangle$ obtained by the simulation relation of Figure 6 is shown. According to Definition 6 , nodes are labeled by four components representing, respectively, states of target, a), b) and c) services. Note that it includes two disconnected components. Obviously, only the one containing the initial state is relevant, as the other one(s) cannot be 

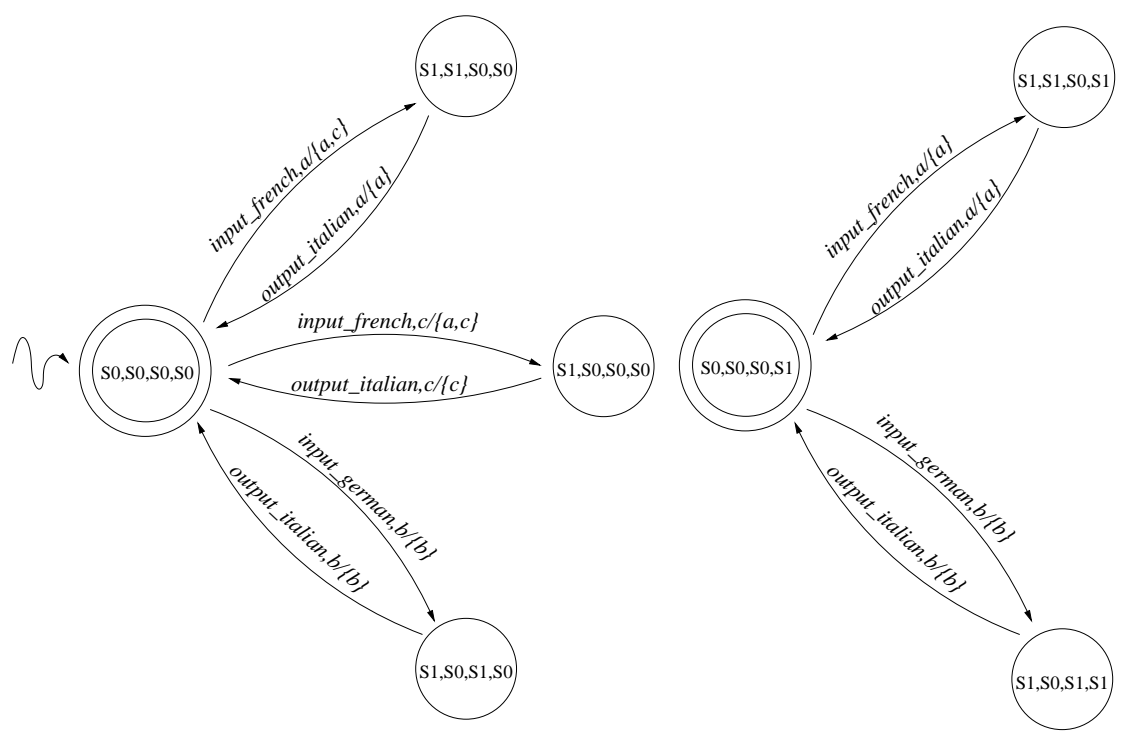

Figure 7: $O G$ for Example 3

reached, all services being initially assumed in their initial state. Edges are labeled by pairs $I / O$, where $I \in A \times[1, \ldots, n]$ and $O \in 2^{[1, \ldots, n]}$, with the following semantics: an edge $e$ connects node $s$ to node $s^{\prime}$ with label $\langle a, i\rangle / O$ iff $\omega_{r}(s, a)=O, i \in O$ and $\delta_{r}(s, a, i)=s^{\prime}$. Starting from this $O G$, several orchestrators can be obtained, depending on the service selected for performing each interaction. Generating an orchestrator corresponds to unfolding an $O G$ and labeling the resulting edges by choosing one among the services proposed by the selection function $\omega_{r}$. For instance, in Figure 8(a) two different orchestrators are reported. Edges are labeled with pairs $a / i$, where $a \in A$ and $i \in[1, \ldots, n]$ represent, respectively, the client requested action and the orch's available service choice.

Now, we show that all generated orchestrators lead to a composition of available services that realizes the client request. Even more importantly, the vice-versa holds: every composition can be obtained by suitably choosing, at each step, one element from those in $O G$ 's selection function $\omega_{r}$. In other words, the maximal simulation virtually contains all compositions. Formally, we have the following theorem.

Theorem 6 Let $\mathcal{C}=\left\{\mathcal{S}_{1}, \ldots, \mathcal{S}_{n}\right\}$ be a community and $S_{t}$ be a target service. Then COMP is a composition of the services in $\mathcal{C}$ realizing $\mathcal{S}_{t}$ if and only if COMP is an orchestrator generated by the orchestrator generator $O G$ for $T S_{t}$ and $T S_{\mathcal{C}}$.

Proof. In order to prove the above theorem we work with composition labelings. A generated orchestrator defines a labeling of $\mathcal{T}_{t}$ by tuples of nodes from $\mathcal{T}_{1} \times \ldots \times \mathcal{T}_{n}$, representing the correspondence between a particular history of the target behavior and those of community services. Such correspondence is strictly related to the history of service assignments, that is, ultimately, to ORCH. Given an orchestrator generator $O G$ for $T S_{t}$ and $T S_{\mathcal{C}}$ and a respective generated orchestrator ORCH, defined as above, an orchestrator labeling OLAB of $\mathcal{T}_{t}^{\mathcal{S}}$ by $\mathcal{T}_{1}^{\mathcal{S}}, \ldots, \mathcal{T}_{n}^{\mathcal{S}}$ is 


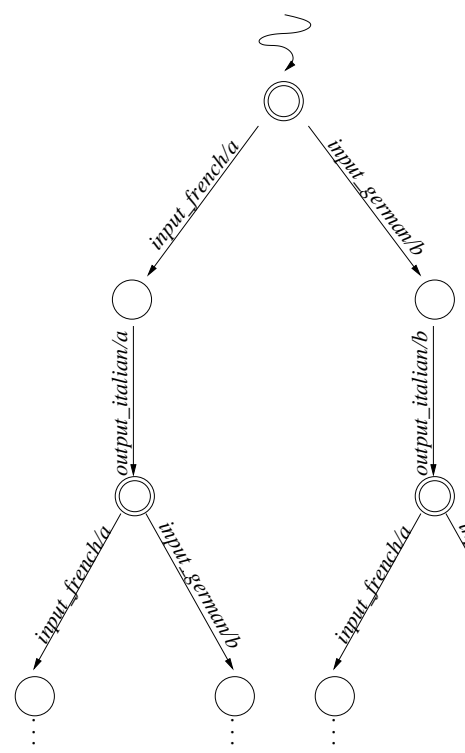

(a)

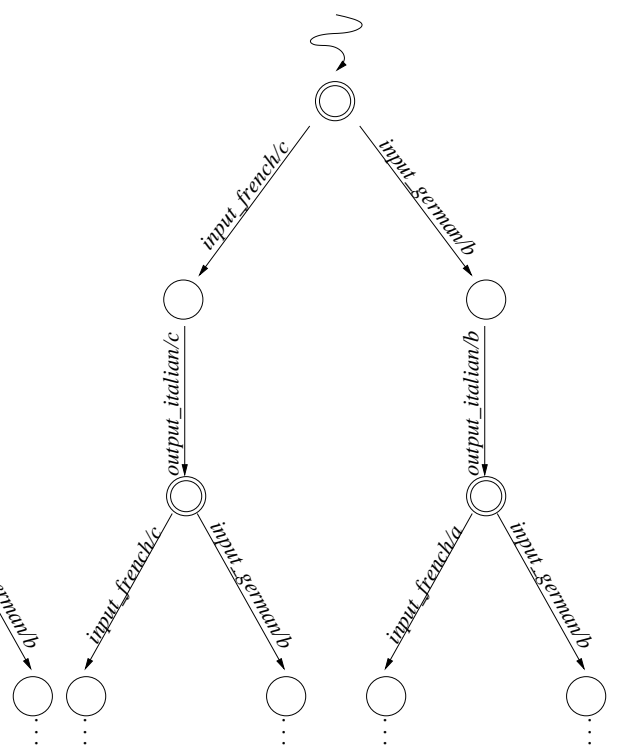

(b)

Figure 8: Two different generated orchestrators for $O G$ of Example 4

defined wrt ORCH as a function OLAB $: \mathcal{T}_{t} \rightarrow \mathcal{T}_{1} \times \ldots \times \mathcal{T}_{n}$, satisfying the following conditions:

- $\operatorname{OLAB}(\varepsilon)=\langle\varepsilon, \ldots, \varepsilon\rangle$

- let $\operatorname{OLAB}(x)=\left\langle x_{1}, \ldots, x_{n}\right\rangle$, for every node $x \in \mathcal{T}_{t}$; if $a \in A$ is such that $x \cdot a \in \mathcal{T}_{t}$ then $\operatorname{OLAB}(x \cdot a)=\left\langle y_{1}, \ldots, y_{n}\right\rangle$, where: $y_{i}=x_{i} \cdot a$ if $\operatorname{OrCH}(x \cdot a)=i$ and $y_{j}=x_{j}$ otherwise.

The following Lemmas are key results for proving our thesis. They state that orchestrator labelings and composition labelings are just different representation of the same entity: a composition.

Lemma 3 If OLAB is an orchestrator labeling of $\mathcal{T}_{t}^{\mathcal{S}}$ by $\mathcal{T}_{1}^{\mathcal{S}}, \ldots, \mathcal{T}_{n}^{\mathcal{S}}$, then OLAB is a composition labeling of $\mathcal{T}_{t}^{\mathcal{S}}$ by $\mathcal{T}_{1}^{\mathcal{S}}, \ldots, \mathcal{T}_{n}^{\mathcal{S}}$.

Proof. We need to show that any orchestrator labeling OLAB fulfills requirements of Definition 1. Let OLAB be an orchestrator labeling of $\mathcal{T}_{t}^{\mathcal{S}}$ by $\mathcal{T}_{1}^{\mathcal{S}}, \ldots, \mathcal{T}_{n}^{\mathcal{S}}$.

1. By definition of orchestrator labeling, $\operatorname{OLAB}(\varepsilon)=\langle\varepsilon, \ldots, \varepsilon\rangle$;

2. By definition of orchestrator labeling, for every node $x \in \mathcal{T}_{t}$, if $\operatorname{OLAB}(x)=$ $\left\langle x_{1}, \ldots, x_{n}\right\rangle$, then for all $a \in A$ such that $x \cdot a \in \mathcal{T}_{t}, \operatorname{OLAB}(x \cdot a)=\left\langle y_{1}, \ldots, y_{n}\right\rangle$, where $y_{i}=x_{i} \cdot a$ if $\operatorname{OrCH}(x \cdot a)=i$ and $y_{j}=x_{j}$ otherwise. Since OlaB is defined wrt an orchestrator $\mathrm{ORCH}$, then $\mathrm{ORCH}(i)$ is defined and identifies the only service capable of performing action $a$. 
Moreover, let $\operatorname{OLAB}(x \cdot a)=\left\langle y_{1}, \ldots, y_{n}\right\rangle$, if $m_{T S_{t}}(x \cdot a)=s_{t}$ and $m_{T S_{i}}\left(y_{i}\right)=s_{i}$ $(i=1, \ldots, n)$ then, from orch and OLAB definitions, it follows that $s_{t} \preceq$ $\left\langle s_{1}, \ldots, s_{n}\right\rangle$.

3. We need to prove that if $x \in \mathcal{T}_{t}$ is final and $\operatorname{OLAB}(x)=\left\langle x_{1}, \ldots, x_{n}\right\rangle$ then all $x_{i}(i=1, \ldots, n)$ are final, as well.

By definition of $m_{T S_{i}}$, a node $x_{i} \in \mathcal{T}_{i}$ is final iff $m_{T S_{i}}\left(x_{i}\right)$ is final for $T S_{i}$ $(i=1, \ldots, n, t)$. Of course, if $x \in \mathcal{T}$ is final then $s_{t}$ also does. Hence, since $s_{t} \preceq\left\langle s_{1}, \ldots, s_{n}\right\rangle$, where $m_{T S_{t}}(x)=s_{t}$ and $m_{T S_{i}}\left(y_{i}\right)=s_{i}(i=1, \ldots, n), s_{i}$ is final for its respective $T S_{i}(i=1, \ldots, n)$ and, consequently, $x_{i}$ is final for its respective execution tree $\mathcal{T}_{i}$.

Lemma 4 If CLAB is a composition labeling of $\mathcal{T}_{t}^{\mathcal{S}}$ by $\mathcal{T}_{1}^{\mathcal{S}}, \ldots, \mathcal{T}_{n}^{\mathcal{S}}$, then CLAB is an orchestrator labeling defined wrt an orchestrator $\mathrm{ORCH}$ generated by the OG for $T S_{t}$ and $T S_{\mathcal{C}}$

Proof. First, observe that, due to Lemma 1, $T S_{\mathcal{C}}$ can simulate $T S_{t}$ and, therefore, the orchestrator generator $O G=\left\langle A,[1, \ldots, n], S_{r}, s_{r}^{0}, \omega_{r}, \delta_{r}, F_{r}\right\rangle$ for $T S_{t}$ and $T S_{\mathcal{C}}$ exists. Now, consider the function $\mathrm{ORCH}: \mathcal{T}_{t} \rightarrow[1, \ldots, n] \cup \perp$, defined as follows:

1. $\operatorname{ORCH}(\varepsilon)=\perp$;

2. $\operatorname{OrCH}(x)=k$ iff there exists a node $x \in \mathcal{T}_{t}$ and an action $a \in A$ such that i) $x \cdot a \in \mathcal{T}_{t}$, ii) $\operatorname{CLAB}(x)=\left\langle x_{1}, \ldots, x_{n}\right\rangle$ and iii) $\operatorname{CLAB}(x \cdot a)=$ $\left\langle x_{1}, \ldots, x_{k} \cdot a, \ldots, x_{n}\right\rangle$ for exactly one $k \in[1, \ldots, n]$.

Referring to Definition 7, we can show ORCH is an orchestrator generated by the $O G$ for $T S_{t}$ and $T S_{\mathcal{C}}$ :

- by definition, $\operatorname{OrCH}(\varepsilon)=\perp$;

- by defining $\sigma_{r}^{\mathrm{ORCH}}(\varepsilon)=s_{r}^{0}$ and, for all $x \cdot a \in \mathcal{T}_{t}, \sigma_{r}^{\mathrm{ORCH}}(x \cdot a)=$ $\delta_{r}\left(\sigma_{r}^{\mathrm{ORCH}}(x), a, \operatorname{ORCH}(x \cdot a)\right)$, we obtain that $\operatorname{ORCH}(x \cdot a) \in \omega\left(\sigma_{r}^{\mathrm{ORCH}}(x), a\right)$ for all $x \cdot a \in \mathcal{T}_{t}$. In fact, if we assume that there exists some $\bar{x} \cdot \bar{a} \in \mathcal{T}_{t}$ such that $\operatorname{ORCH}(\bar{x} \cdot \bar{a}) \notin \omega_{r}\left(\sigma_{r}^{\mathrm{ORCH}}(\bar{x}), \bar{a}\right)$, then CLAB would not be a composition labeling, since there would exist no $k \in[1, \ldots, n]$ such that $\operatorname{CLAB}(\bar{x})=\left\langle\bar{x}_{1}, \ldots, \bar{x}_{n}\right\rangle$ and $\operatorname{CLAB}(\bar{x} \cdot \bar{a})=\left\langle\bar{x}_{1}, \ldots, \bar{x}_{k} \cdot \bar{a}, \ldots, \bar{x}_{n}\right\rangle$.

Finally, we need to show that CLAB is defined with respect to ORCH, according to the definition of orchestrator labeling, but this straightforward follows from requirement 2 of ORCH's construction.

With this lemmas in place, we can finally go back to Theorem 6 . Indeed the thesis of the theorem directly follows from Lemmas 3 and 4 , by recalling the equivalence between composition and composition labeling (see Definitions 1 and 2).

As already pointed out, Theorem 6 yields that, given an $O G$, by nondeterministically choosing a service among those proposed by the selection function 
$\omega_{r}$, we obtain all and only the orchestrators $O G$ generates. Interestingly, orchestrators are not required to be built before a client starts interacting with the community, but can be generated just-in-time, as client issues action requests. Formally, given an orchestrator generator $O G$ for $T S_{t}$ and $T S_{\mathcal{C}}$, defined as in Definition 6 , a just-in-time (generated) orchestrator is a function JIT_ORCH : $\mathcal{T}_{t} \rightarrow[1, \ldots, n] \cup \perp$, inductively defined as follows:

- JIT_ORCH $(\varepsilon)=\perp$;

- if $x \cdot a \in \mathcal{T}_{t}$, then JIT_ORCH $(x \cdot a)=\operatorname{choose}\left(\omega_{r}\left(\sigma_{r}^{\mathrm{JIT}}{ }^{\mathrm{ORCH}}(x), a\right)\right)$, where $\sigma_{r}^{\mathrm{JIT} \_ \text {ORCH }}: \mathcal{T}_{t} \rightarrow S_{r}$ is the mapping function between nodes of $\mathcal{T}_{t}$ and corresponding states of $S_{r}$ defined as in Definition 7 and choose stands for a choice function that chooses one element among those returned by $\omega_{r}\left(\sigma_{r}^{\mathrm{JIT}} \_\mathrm{ORCH}(x), a\right)$.

Obviously, with appropriate choice functions for choose, one can get all possible generated orchestrators. But, the point of the definition above is that one can delay the choice performed by choose till run-time, where one can take into account information on the actual state, cost, etc., of the execution of actions by the various services. This gives a great flexibility to the orchestrator, which, in a sense, can "switch" composition on the go as needed. As a result, this work can be seen as providing formal bases for research work aimed at developing ambient-aware compositions that are fully reactive to events occurring during execution.

\section{Conclusion}

In this paper we have explored the possibility of basing service composition on the notion of simulation. We have seen that by using simulation, we are able to virtually compute all possible compositions at once, and that this opens the possibility of devising composition in a just-in-time fashion.

The tight connection between service composition and simulation discussed here, allows us to transfer well developed techniques for computing simulation, such as those in $[20,32,13]$ to service composition. Interestingly, also known result from service composition can be transferred to simulation. In particular, the EXPTIMEcompleteness of service composition, allows us to say that checking simulation from a single deterministic transition system to the asynchronous product of to $n$ deterministic transition systems is an EXPTIME-complete problem. Notably, this closes a long standing open problem in the simulation literature.

\section{References}

1. V. Agarwal, G. Chafle, K. Dasgupta, N. M. Karnik, A. Kumar, S. Mittal, and B. Srivastava. Synthy: A system for end to end composition of web services. $J$. Web Sem., 3(4):311-339, 2005.

2. G. Alonso, F. Casati, H. Kuno, and V. Machiraju. Web Services. Concepts, Architectures and Applications. Springer, 2004.

3. D. Berardi. Automatic Composition Services: Models, Techniques and Tools. PhD thesis, Università degli Studi di Roma - La Sapienza, 2005. 
4. D. Berardi, D. Calvanese, G. De Giacomo, R. Hull, and M. Mecella. Automatic Composition of Transition-based Semantic Web Services with Messaging. In Proc. of VLDB 2005, 2005.

5. D. Berardi, D. Calvanese, G. De Giacomo, M. Lenzerini, and M. Mecella. Automatic Composition of e-Services that Export their Behavior. In Proc. of ICSOC 2003, pages $43-58,2003$.

6. D. Berardi, D. Calvanese, G. De Giacomo, M. Lenzerini, and M. Mecella. Synthesis of Underspecified Composite e-Services based on Automated Reasoning. In Proc. of ICSOC 2004, pages 105-114, 2004.

7. D. Berardi, D. Calvanese, G. De Giacomo, M. Lenzerini, and M. Mecella. Automatic service composition based on behavioral descriptions. International Journal of Cooperative Information Systems, 14(4):333 - 376, 2005.

8. D. Berardi, G. De Giacomo, and M. Mecella. Basis for automatic service composition. Tutorial at $W W W 2005,2005$.

9. T. Bultan, X. Fu, R. Hull, and J. Su. Conversation Specification: A New Approach to Design and Analysis of E-Service Composition. In Proc. of WWW 2003, 2003.

10. G. De Giacomo. Composition synthesis of web services composition synthesis of web services. Talk at Daghstuhl Seminar on Synthesis and Planning, 2005.

11. G. De Giacomo and M. Mecella. Service composition. Tutorial at ICSOC 2004, 2004.

12. G. De Giacomo and S. Sardiña. Automatic synthesis of new behaviors from a library of available behaviors. In Proc. of IJCAI 200\%, pages 1866-1871, 2007.

13. R. Gentilini, C. Piazza, and A. Policriti. From bisimulation to simulation: Coarsest partition problems. J. Autom. Reasoning, 31(1):73-103, 2003.

14. C. E. Gerede, R. Hull, O. H. Ibarra, and J. Su. Automated composition of e-services: lookaheads. In Proc. of ICSOC 2004, pages 252-262, 2004.

15. C. E. Gerede, O. H. Ibarra, B. Ravikumar, and J. Su. Online and minimum-cost ad hoc delegation in e-service composition. In Proc. of the IEEE 2005 IEEE International Conference on Services Computing (SCC'05), pages 103-112, 2005.

16. M. Ghallab, D. Nau, and P. Traverso. Automated Planning: Theory and Practice. Morgan Kauffman, 2004.

17. G. Grahne and V. Kiricenko. Process mediation in an extended roman model. In Proceedings of the First International Workshop on Mediation in Semantic Web Services (MEDIATE 2005), pages 17 - 33, 2005.

18. A. Y. Halevy. Answering Queries Using Views: A Survey. VLDB Journal, 10(4):270-294, 2001.

19. D. Harel, D. Kozen, and J. Tiuryn. Dynamic Logic. The MIT Press, 2000.

20. M. R. Henzinger, T. A. Henzinger, and P. W. Kopke. Computing simulations on finite and infinite graphs. In Proc. of FOCS 1995, pages 453-462, 1995.

21. R. Hull, M. Benedikt, V. Christophides, and J. Su. E-Services: a Look Behind the Curtain. In Proc. of PODS 2003, pages 1-14, 2003.

22. M. Lenzerini. Data Integration: A Theoretical Perspective. In Proc. of PODS 2002, pages 233-246, 2002.

23. S. A. McIlraith and T. C. Son. Adapting Golog for Composition of Semantic Web Services. In Proc. of KR 2002, pages 482-496, 2002.

24. B. Medjahed, A. Bouguettaya, and A. K. Elmagarmid. Composing Web Services on the Semantic Web. VLDB Journal, 12(4):333-351, 2003. 
25. M. Michalowski, J. L. Ambite, C. A. Knoblock, S. Minton, S. Thakkar, and R. Tuchinda. Retrieving and Semantically Integrating Heterogeneous Data from the Web. IEEE Intelligent Systems, 19(3):72-79, 2004.

26. R. Milner. An algebraic definition of simulation between programs. In Proc. of IJCAI 1971, pages 481-489, 1971.

27. A. Muscholl and I. Walukiewicz. A Lower Bound on Web Services Composition. In Proc. of the 10th Int. Conf. on Foundations of Software Science and Computation Structures (FoSSaCS 2007), volume 4423 of LNCS. Springer, 2007.

28. M. Pistore, P. Traverso, P. Bertoli, and A. Marconi. Automated Synthesis of Composite BPEL4WS Web Services. In Proc. of ICWS 2005, 2005.

29. A. Pnueli and R. Rosner. On the Synthesis of a Reactive Module. In Proc. of POPL 1989, pages 179-190, 1989.

30. S. Sardiña, F. Patrizi, and G. De Giacomo. Automatic synthesis of a global behavior from multiple distributed behaviors. In Proc. of AAAI 2007, pages 1063-1069, 2007.

31. S. K. Shukla, H. B. H. III, D. J. Rosenkrantz, and R. E. Stearns. On the complexity of relational problems for finite state processes. In Proc. of ICALP 1996, pages 466-477, 1996.

32. L. Tan and R. Cleaveland. Simulation revisited. In Proc. of TACAS 2001, pages 480-495, 2001.

33. W. Thomas. Infinite Games and Verification. In Proc. of CAV 2002, 2002.

34. J. D. Ullman. Information Integration using Logical Views. Theoretical Computer Science, 239(2):189-210, 2000.

35. M. Y. Vardi. An Automata-Theoretic Approach to Fair Realizability and Synthesis. In Proc. of CAV 1995, 1995.

36. J. Yang and M. P. Papazoglou. Service Components for Managing the Life-Cycle of Service Compositions. Information Systems, 29(2):97-125, 2004. 